\title{
FORM FOLLOWS AVAILABILITY - DESIGNING STRUCTURES THROUGH REUSE
}

\author{
Jan BRÜTTING ${ }^{1}$, Gennaro SENATORE ${ }^{2}$ and Corentin FIVET ${ }^{1}$ \\ ${ }^{1}$ Structural Xploration Lab, Swiss Federal Institute of Technology Lausanne (EPFL), Switzerland \\ Corresponding author: jan.bruetting@epfl.ch \\ ${ }^{2}$ Applied Computing and Mechanics Lab, Swiss Federal Institute of Technology Lausanne (EPFL), Switzerland
}

Editor's Note: The first author of this paper is one of the four winners of the 2019 Hangai Prize, awarded for outstanding papers that are submitted for presentation and publication at the annual IASS Symposium by younger members of the Association (under 30 years old). It is re-published here with permission of the editors of the proceedings of the IASS Symposium 2019: "Form and Force" held in October 2019 in Barcelona, Spain.

DOI: https://doi.org/10.20898/j.iass.2019.202.033

\section{ABSTRACT}

This work proposes a new direction in structural design: the synthesis of structures through the reuse of elements. Reusing structural elements reduces the environmental impacts of building structures because it avoids sourcing new material, it reduces waste and it requires little energy. Designing structures from reused elements is unlike conventional structural design because stock element availability is a design input. In other words, structures must be designed subject to availability of given element characteristics such as length and cross-section type, which have a major influence on the optimal structure layout and form. In this new paradigm structural form follows availability. In this work new computational methods for the synthesis of reticular structures through reuse are formulated to address two scenarios: a) reuse of reclaimed elements from a given stock, and b) design of an element stock which is used as a kit of parts to build diverse structures. Case studies are presented to demonstrate the potential of the proposed methods. It is shown that structures produced by these methods have a significantly lower environmental impact than minimum weight structures made of new elements.

Keywords: Structural design, discrete optimization, geometry optimization, mixed-integer linear programming, reuse, Life Cycle Assessment, environmental impact

\section{INTRODUCTION}

\subsection{Reuse in structural design}

Designing structures through reuse is a challenging task because "size and length of the available members will then determine the spans and spacing possible in the new structure [...]" [1]. In other words, the design process is reversed because mechanical and geometric properties of available elements have a major influence on the structural layout. This new paradigm where form follows availability [2] is not yet supported by design guidelines nor by established computational tools. Figure 1 illustrates the two methods outlined in this paper: a) designing structures from a stock of available elements; b) designing a stock of elements, which is used as a kit of parts to build different structures. Both these design approaches enable the reuse of structural components for multiple service lives. These approaches support circular economy principles through reducing raw material usage, waste generation and energy consumption, thus mitigating environmental impacts of load-bearing structures.

(a)

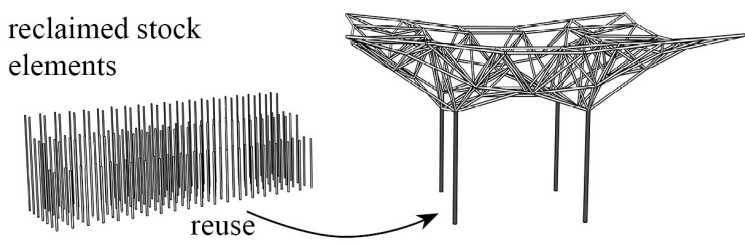

(b)

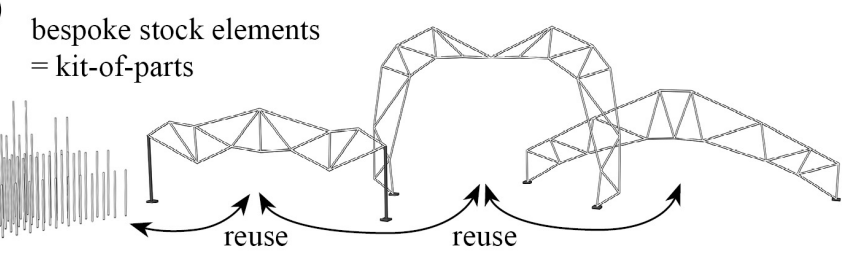

Figure 1: Designing structures through reuse: (a) a spatial truss made from a stock of reclaimed elements, (b) three trusses made from one kit of parts 


\subsection{Stock-constrained structural optimization}

Structural optimization with stock constraints has received little attention so far. The sizing optimization of plane frames of fixed topology from a stock of onetime available cross sections has been presented in [3]. In [3] evolutionary algorithms have been employed for weight optimization but without accounting for the availability of element lengths. Strategies based on heuristic algorithms have been developed to form-fit a stock of wood logs to statically determinate trusses [4]. Different to previous works, the formulations presented in this paper are based on deterministic combinatorial optimization that guarantees a globally optimal element assignment.

With respect to designing structures for multiple purposes, Nadir et al. [5] have studied the reconfiguration of bars within a planar truss, in order to react to changing load actions. Tugilimana et al. [6] considered sizing optimization, spatial rearrangement and reuse of unit-cells in modular bridges. Basso et al. [7] optimized the geometry of a free-form grid structure for a defined number of elements of different length with the objective to simplify fabrication. Different to previous works, the kit-of-parts optimization method presented in this paper 1) considers stocks of individual elements with varying cross-sections and lengths, 2) carries out element assignment, topology, and geometry optimization, and 3) minimizes structural weight, environmental impacts and the number of unshared elements between structures made from a common kit of parts.

\subsection{Outline}

Section 2 outlines the formulation for the design of structures with reused elements, which is based on discrete structural optimization [8]. Section 2.1 focuses on assignment and topology optimization of reticular structures subject to stock constraints. This method has been previously presented in [9] for 2D configurations and it is here extended to $3 \mathrm{D}$ structures. Section 2.2 gives a new formulation to obtain an optimal stock of elements (i.e. a kit of parts) that can be used to build different structures. Sections 2.3 to 2.5 outline the main aspects of the structural and geometric optimization techniques as well as of the Life Cycle Assessment employed in this study. Section 3 presents the application of the optimization methods to case studies.

\section{METHODS}

\subsection{Assignment and topology optimization (P)}

In the following, stock components are named elements while components of a truss structure are named members. Formulation $(\mathrm{P})$ is the optimum assignment $\boldsymbol{T}$ of a given stock of elements into a predetermined structural layout (also called ground structure) comprising $m$ members. The objective (Eq. 1) of (P) is the minimization of the structure embodied energy (section 2.5). It is assumed that stock elements are collected in $g$ groups of identical length and cross section. The use of one element from stock group $j$ at member position $i$ in the ground structure is represented by the entry $t_{i, j}=1$ in the binary assignment matrix $\boldsymbol{T} \in\{0,1\}^{m \times g}$. If element $j$ is not assigned at position $j$ then $t_{i, j}=0$. A group of 'zero-members' can be added to the stock to carry out topology optimization. Eq. (2) enforces the assignment of only one element from the stock to any position in the structure. Eq. (3) constrains the maximum number of element assignments to $n_{j}$ available elements per group. Eq. (4) constrains the lengths $\boldsymbol{l} \in \mathbb{R}^{g}$ of assigned elements to be longer or equal than the distance $\overline{\boldsymbol{l}} \in \mathbb{R}^{m}$ between the nodes of the corresponding truss positions.

$$
\min _{T} f(\boldsymbol{T})
$$

$$
\begin{aligned}
\text { s.t.: } \quad & \sum_{j=1}^{\mathrm{g}} t_{i, j}=1 \quad \forall i=1 \ldots m \\
& \sum_{i=1}^{m} t_{i, j} \leq n_{j} \forall j=1 \ldots g
\end{aligned}
$$$$
\bar{l} \leq \boldsymbol{T l}
$$

\subsection{Stock optimization (S)}

Formulation (S) is the optimization of the stock configuration (i.e. kit of parts) such that its elements are reused to build $\mathcal{R}$ different structures. This process can be thought of as the "inverse" of $(\mathrm{P})$. The first term of the multi-objective function (Eq. 5) minimizes the total number of stock elements $N_{t o t}$, which in turn maximizes element reuse. The other two terms minimize cut-off waste $W^{r}$ and the weight $M^{r}$ of each structure $r \in \mathcal{R}$. The objective function can be weighted to bias the optimization towards a specific objective. In this work, weighting factors $w_{1}$ $=1, w_{2}=0.1$ and $w_{3}=0.2$ have proven to give best results and are used in the following. For a 
predefined set $\mathcal{H}$ of standard cross sections, the number of elements $n_{j}$ and lengths $l_{j}$ for each cross section $j \in \mathcal{H}$ are design variables. The binary variable $z_{j}$ determines whether elements with cross section $j$ and length $l_{j}$ are part of the optimum stock $\left(z_{j}=1\right)$ or not $\left(z_{j}=0\right)$. For each element type at most $n_{j}$ elements can be assigned to a structure $r$ (Eq. 7). An upper bound $z^{\max }$ constrains the variables $z_{j}$ to obtain a maximum number of element types (i.e. cross sections and lengths) in the stock (Eq. 8). Eq. (9) constrains the member lengths $\overline{\boldsymbol{l}}^{r}$ within a defined range $\Delta$ to the stock element lengths $l$. $\Delta$ is used here as a tolerance measure in order to relax the optimization problem. In practice $\Delta$ is the difference between the length of a kit-of-parts element and the distance of the nodes within which the element has to be fitted. This difference could be compensated through adjustable joints.

$$
\begin{gathered}
\min _{T, \boldsymbol{n}, \boldsymbol{l}, \boldsymbol{z}} w_{1} N_{t o t}+w_{2} W_{t o t}+w_{3} M_{t o t} \\
=w_{1} \sum_{j \in \mathcal{H}} n_{j}+w_{2} \sum_{r \in \mathcal{R}} W^{r}+w_{3} \sum_{r \in \mathcal{R}} M^{r}
\end{gathered}
$$

s.t.:

$$
\begin{gathered}
\sum_{j \in \mathcal{H}} t_{i, j}^{r}=1 \forall i=1 \ldots m^{r} ; r \in \mathcal{R} \\
\sum_{i=1}^{m^{r}} t_{i, j}^{r} \leq z_{j} n_{j} \forall j \in \mathcal{H} ; r \in \mathcal{R} \\
\sum_{j \in \mathcal{H}} z_{j} \leq z^{\max } \\
\boldsymbol{T}^{r} \boldsymbol{l}-\Delta \leq \overline{\boldsymbol{l}}^{r} \leq \boldsymbol{T}^{r} \boldsymbol{l} \forall r \in \mathcal{R}
\end{gathered}
$$

\subsection{Structural optimization}

Since the problems outlined in sections 2.1 and 2.2 are formulated as Mixed-Integer Linear Programs (MILP) in standard form, they can be solved to global optimality via branch-and-bound techniques. Structural optimization is included into these formulations through the simultaneous analysis and design approach [10]. Force equilibrium, geometric compatibility, stress and displacement constraints are added to $(\mathrm{P})$ or $(\mathrm{S})$ as linear equality or inequality constraints. Self-weight and member buckling are also considered. For details concerning the structural optimization part of these formulations, the reader is referred to [9].

\subsection{Geometry optimization}

Geometry optimization is carried out in sequence with $(\mathrm{P})$ or $(\mathrm{S})$ over a predefined number of iterations in order to improve the structural design. When considering $(\mathrm{P})$, geometry optimization is carried out in two steps. In the first step the objective is to minimize compliance (i.e. maximize the stiffness). This way the structural shape is optimized allowing the assignment of smaller cross section elements. Subsequently, the objective of geometry optimization is changed to minimize cut-off waste.

When considering (S), geometry optimization is carried out to improve fitting between the kit of parts and the structures. For this reason, the stock element lengths $\boldsymbol{l}$ are included as design variables. The tolerance $\Delta$ is decreased at each iteration until all elements in the kit of parts can be assigned using their full length $(\Delta=0$, i.e. no cut-off and waste).

\subsection{Life Cycle Assessment}

Life Cycle Assessment (LCA) is carried out to quantify embodied energy and carbon equivalence. When considering component reuse (P), this assessment accounts for impacts related to the supply of stock elements through selective deconstruction as well as transport to the building site. The embodied energy $E E_{R}$ and carbon equivalence $E C_{R}$ of structures made from reused elements is here defined as a function of structural mass $M$ and cutoff waste $W$ :

$$
\begin{gathered}
E E_{R}=3.245 \frac{\mathrm{MJ}}{\mathrm{kg}} \mathrm{M}+3.235 \frac{\mathrm{MJ}}{\mathrm{kg}} \mathrm{W} \\
E C_{R}=0.277 \frac{\mathrm{kgCO} \mathrm{C}_{2} \mathrm{eq}}{\mathrm{kg}} M+0.276 \frac{\mathrm{kgCO} \mathrm{C}_{2} \mathrm{eq}}{\mathrm{kg}} \mathrm{W}
\end{gathered}
$$

The coefficients in Eqs. (10) and (11) are derived from the same assumptions as taken in [9].

To benchmark the environmental savings obtained through reuse against newly produced elements, production methods involving primary and secondary (recycled) steel are considered. The embodied energy $E E_{N}$ and carbon equivalence $E C_{N}$ for conventionally produced steel profiles and their transport to the site are $13.227 \frac{\mathrm{MJ}}{\mathrm{kg}}$ and $0.925 \frac{\mathrm{kgCO}}{\mathrm{kg}}$ respectively. Production of connection elements is assumed similar in both reuse and new cases and is therefore omitted from the assessment. 


\section{RESULTS}

3.1. Optimization of a 2D truss under stock constraints

Figure 2(a) shows a truss ground structure with $\mathrm{m}=41$ possible bar positions and a span of $1=20.00 \mathrm{~m}$. A dead load of $1.00 \mathrm{kN} / \mathrm{m}$ and a live load of $5.00 \mathrm{kN} / \mathrm{m}$ are applied to the top chord nodes. The optimization is subject to ultimate (ULS) and serviceability limit state (SLS). For the SLS load combination, displacements are limited to $l / 300$. In the geometry optimization step all nodes are constrained within a region of $\pm 3.00 \mathrm{~m}$ to prevent self-overlapping. The position of the roller support is bounded horizontally to maintain a minimum span of $20.00 \mathrm{~m}$.

(a)

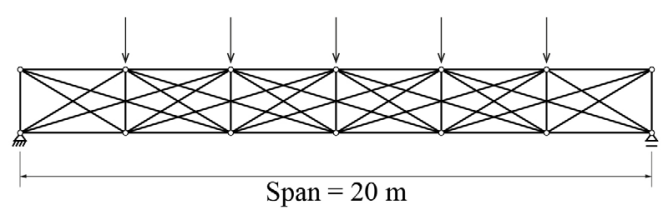

(b)

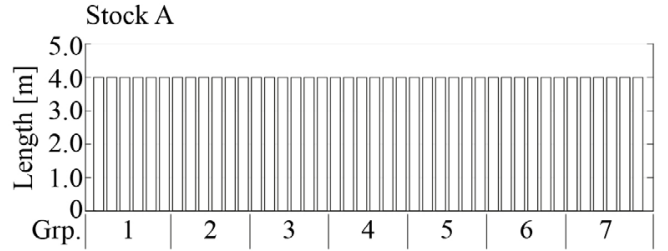

(c)

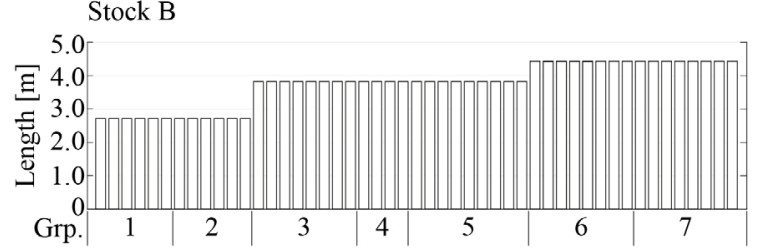

(d)

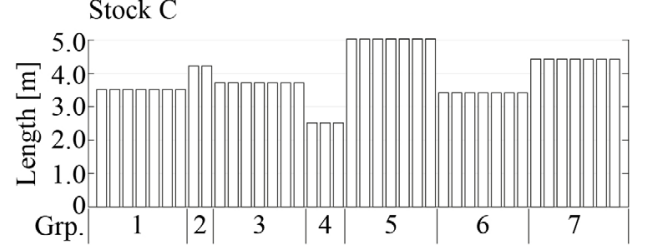

Figure 2: Optimization setup: a) ground structure and loading, b) -d) Stocks $A, B$ and $C$

Three stock configurations $\mathrm{A}, \mathrm{B}$, and $\mathrm{C}$ considered in this study are illustrated in Figure 2(b)-(d). All three stocks consist of circular hollow sections (CHS) of dimensions taken from EN 10210. For each stock, seven element groups with cross section sizes $33.7 \times 3.2,42.4 \times 4,48.3 \times 5,60.3 \times 4,76.1 \times 5$, $88.9 \times 10$, and $139.7 \times 5$ are available. In stock A all elements have $4.00 \mathrm{~m}$ length. In stock B three (a)

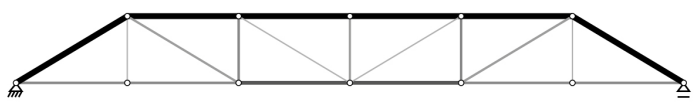

(b)

Stock A

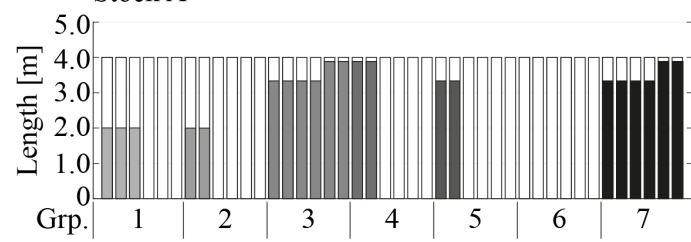

Figure 3: Optimal assignment and topology from Stock A with input geometry

(a)

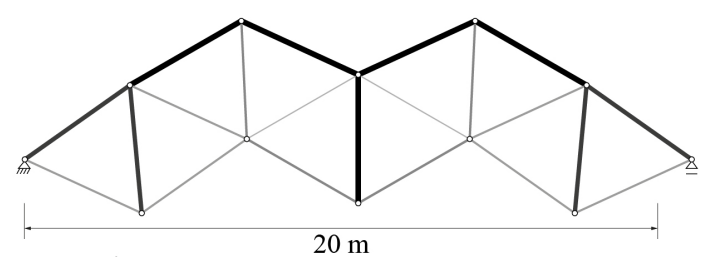

Stock A

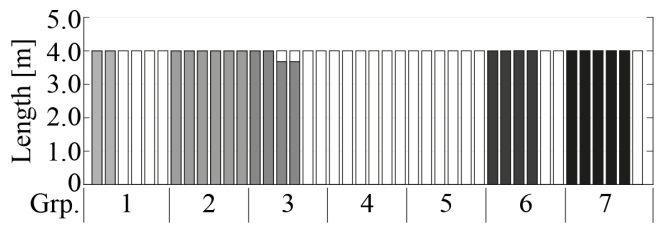

(b)
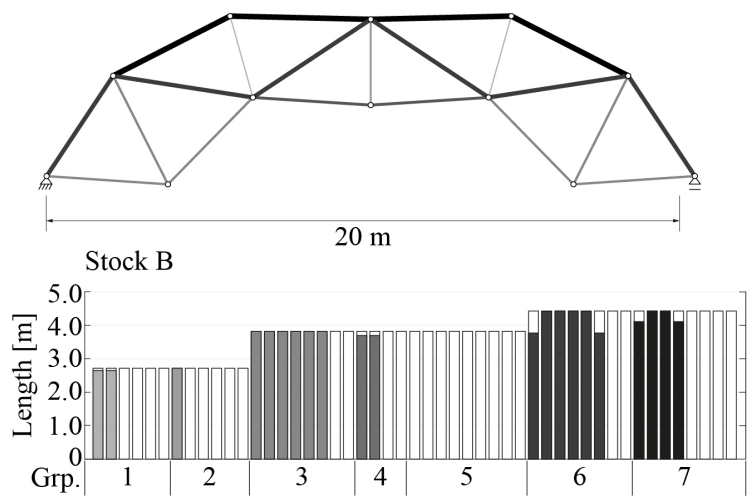

(c)
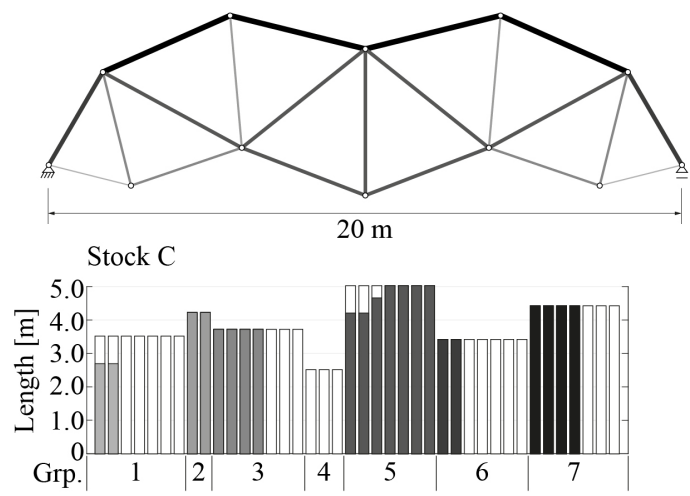

Figure 4: Optimum designs for stocks $A, B$ and $C$ after geometry optimization 
different element lengths $(2.70,3.80,4.40 \mathrm{~m})$ are available. Such element length configurations might for instance originate from disassembled Warren or Pratt trusses. Stock C consists of elements with random lengths between $2.5 \mathrm{~m}$ and $5.0 \mathrm{~m}$. All stock elements are assumed to be standard grade steel S235. However, for reused elements a material safety factor of $90 \%$ is assumed to account for uncertainties on the element capacity and material degradation.

Figure 3 shows the optimum assignment solution with input geometry for Stock A (i.e. the first solution of (P)). Figure 3(b) shows a bar chart representing the stock and element assignment. White bars represent stock elements or cut-off. Greyscale bars map the truss members onto selected stock elements and indicate the element group. The darker the shade the bigger the cross section area of the element. For brevity and because they have identical geometry, the solutions obtained from stock $\mathrm{B}$ and $\mathrm{C}$ are not illustrated.

Figure 4 shows the optimal configurations after geometry optimization to minimize compliance and cut-off waste. Using stock A results in a structure that consists of equilateral triangles, Figure 4(a). Only two elements of group 3 require cutting as shown in the corresponding bar chart. The elements of groups 4 and 5 are not used because either the assignment of smaller sections was possible (groups 1 to 3 ) or larger sections were required (groups 6 and 7). Using stock B results in a structure with an arched top chord which makes use of all available element lengths, Figure 4(b). In the case of stock $C$ all except five stock elements are placed without requiring cutting, Figure 4(c). In all cases, elements with larger cross sections are assigned to the top chord because of member buckling constraints.

A weight-optimized structure made of new steel elements assuming an infinite availability of all standard sections in EN 10210 is taken as a benchmark, Figure 5. This benchmark is obtained through a sequential discrete sizing and geometry optimization (see section 2). In this case compliance minimization is the sole objective of the geometry optimization steps.

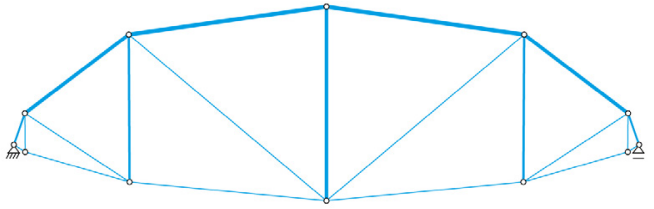

Figure 5: Minimum weight benchmark made from new elements
It is clear that, due to the stock constraints, the reuse cases do not have a similar geometry to the minimum weight solution shown in Figure 5.

Table 1 and 2 give a summary of results for the solutions obtained without and with geometry optimization, respectively. Because the number of small cross sections is limited in the stocks, the systems with reused elements are oversized compared to minimum weight solutions ('New'). A higher member force capacity utilization (util.) is obtained for the minimum weight solution. However, reusing reclaimed structural elements results in a significant reduction of embodied energy and carbon equivalence: between $46 \%$ to $64 \%$ for the solutions obtained without geometry optimization and between 36 and 59\% for the geometry-optimized structures.

Table 1: Results - 2D case studies with input geometry

\begin{tabular}{|c|c|c|c|c|c|c|c|c|}
\hline Stock & Case & Mass & Waste & Cut & $\begin{array}{l}\text { mean } \\
\text { util. } \\
{[-]}\end{array}$ & $\begin{array}{l}\max \\
\text { util. } \\
{[-]}\end{array}$ & {$[\mathrm{MJ}]$} & {$\left[\mathrm{kgCO}_{2 \mathrm{eq}}\right]$} \\
\hline A & (P) & 468 & 84 & 21 & $68 \%$ & $88 \%$ & 1'790 & 150 \\
\hline B & (P) & 489 & 98 & 21 & $62 \%$ & $88 \%$ & 1'900 & 160 \\
\hline C & (P) & 605 & 97 & 25 & $59 \%$ & $88 \%$ & 2'280 & 190 \\
\hline New & & 380 & - & - & $82 \%$ & $100 \%$ & 5'030 & 350 \\
\hline
\end{tabular}

Table 2: Results - 2D case studies after sequential assignment and geometry optimization

\begin{tabular}{|c|c|c|c|c|c|c|c|}
\hline \multirow[t]{2}{*}{ Stock Case } & \multirow{2}{*}{$\begin{array}{l}\text { Mass } \\
{[\mathrm{kg}]}\end{array}$} & \multicolumn{3}{|c|}{$\begin{array}{l}\text { Waste Cuts mean } \\
\text { util. }\end{array}$} & \multirow{2}{*}{$\begin{array}{l}\max \\
\text { util. } \\
{[-]}\end{array}$} & \multirow{2}{*}{$\begin{array}{l}\text { EE } \\
{[\mathrm{MJ}]} \\
\end{array}$} & \multirow{2}{*}{$\begin{array}{l}\mathrm{EC} \\
{\left[\mathrm{kgCO}_{2 \mathrm{eq}}\right]}\end{array}$} \\
\hline & & {$[\mathrm{kg}]$} & {$[-]$} & {$[-]$} & & & \\
\hline (P) $+\mathrm{G}_{\mathrm{opt}}$ & 497 & 3 & 2 & $74 \%$ & $90 \%$ & $1 ' 630$ & 140 \\
\hline (P) $+\mathrm{G}_{\text {opt }}$ & 534 & 19 & 8 & $56 \%$ & $90 \%$ & $1^{\prime} 790$ & 150 \\
\hline$(\mathrm{P})+\mathrm{G}_{\mathrm{opt}}$ & 640 & 21 & 5 & $45 \%$ & $88 \%$ & 2'150 & 180 \\
\hline New & 298 & - & - & $72 \%$ & $98 \%$ & 3'940 & 280 \\
\hline
\end{tabular}

\subsection{Optimization of a 3D space truss under} stock constraints

Figure 6(a) shows a roof structure of $18.00 \mathrm{~m}$ width and $\mathrm{m}=200$ members. The vertical columns in Figure 6 represent the four vertical supports but are not part of the optimization. A distributed load of $2.25 \mathrm{kN} / \mathrm{m}^{2}$ is applied to the top chord nodes. In the geometry optimization step the perimeter nodes are constrained to maintain a minimum $18 \times 18 \mathrm{~m}$ footprint. The structure is optimized subject to the same stocks as in section 3.1 albeit with an increased element availability. The availability of elements in stock A is six times that of the 2D case; stocks B and $\mathrm{C}$ have 40 elements per group. 
In this case study, the topology is invariant, i.e. one stock element is assigned to each position. A symmetric assignment of elements $(\mathrm{XZ}$ and $\mathrm{YZ}$ plane) is enforced to reduce the number of binary assignment variables. In addition, geometric compatibility and nodal displacements are omitted (plastic design). These simplifications reduce computational complexity, which is important for this case study as it contains a large number of members. This way it is possible to solve problem (P) to global optimality within a reasonable computation time ( $<1 \mathrm{~h}$ on an Intel i7-6820HQ 2.70 $\mathrm{GHz} \mathrm{CPU})$.

Figure 6(b) - (d) shows the optimized structural systems for stocks A, B and C respectively. The weight-optimized structure made of new elements is illustrated in Figure 6(e). A different shade of grey is used to represent the seven stock element groups (section 3.1). The top views show an increase of footprint for the structures obtained through reuse. As stock A consists of equal length elements, the resulting structure geometry remains regular. In the case of stock B and C more irregular geometries are obtained. The geometry optimization successfully reduces waste and cutting of stock elements (Table 3). Most elements can be reused at their full length. For all three stocks between one fourth and one third out of the total number of members (200) require cutting. Similar to the $2 \mathrm{D}$ case study in section 3.1 , reusing structural elements results in a significant reduction of embodied carbon and energy, even though these systems have a higher mass and lower mean capacity utilization with respect to the weightoptimized solution obtained from new elements.

(a)
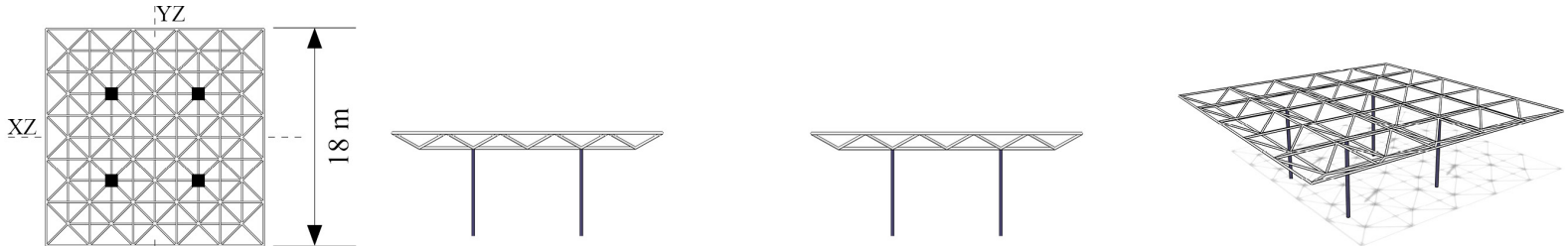

(b)

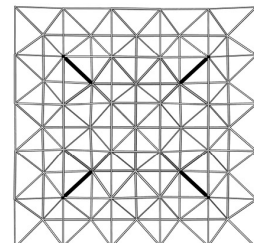

Stock A
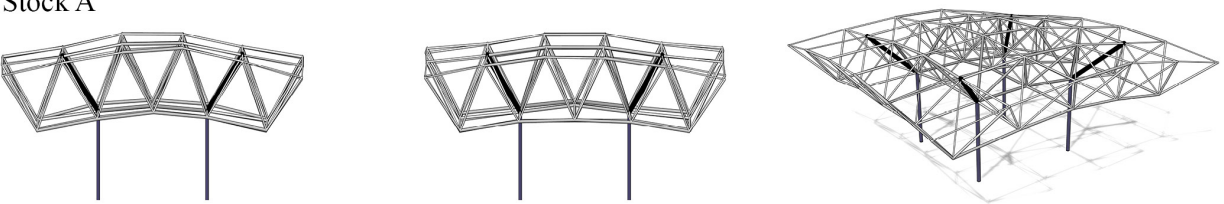

(c)
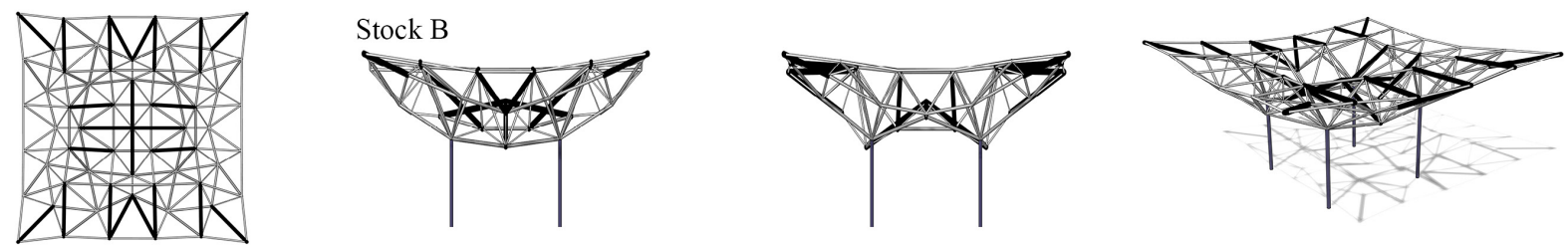

(d)
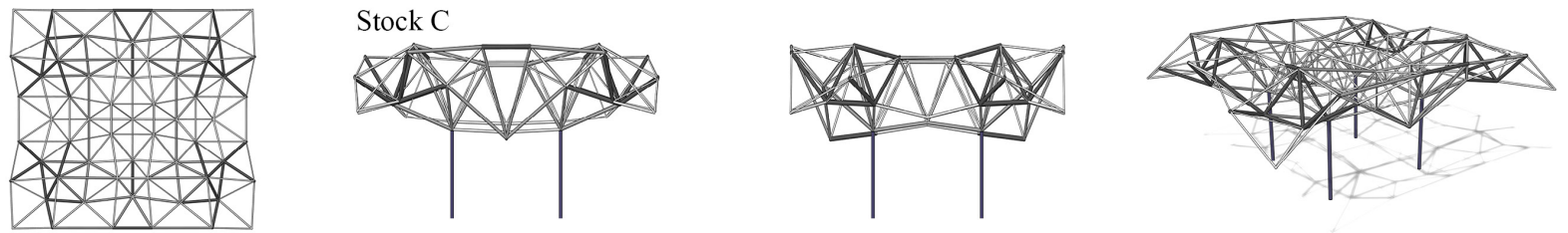

(e)
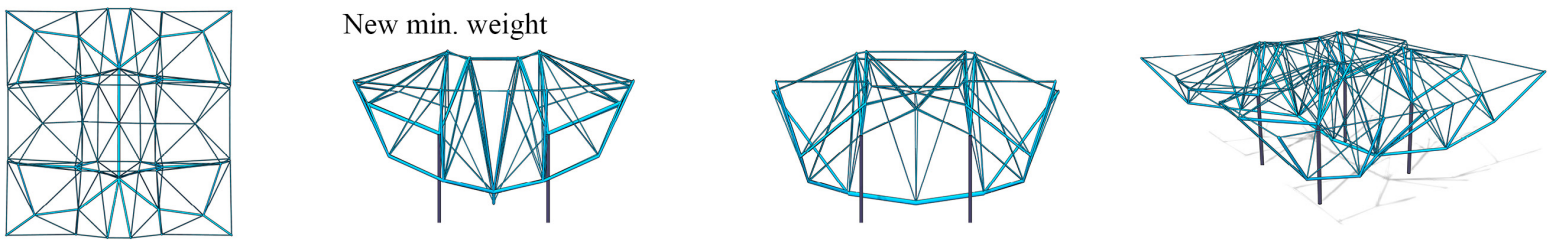

Figure 6: Optimum 3D designs: (a) system definition, (b-d) optimal solutions from stocks $A$, $B$, and $C$, (e) minimum weight solution with new elements 
Table 3: Summary of results for 3D case studies

\begin{tabular}{|c|c|c|c|c|c|c|c|c|}
\hline Stock & Case & $\begin{array}{l}\text { Mass } \\
{[\mathrm{kg}]}\end{array}$ & $\begin{array}{l}\text { Waste } \\
{[\mathrm{kg}]}\end{array}$ & $\begin{array}{l}\text { Cuts } \\
{[-]}\end{array}$ & $\begin{array}{l}\text { mean util. } \\
{[-]}\end{array}$ & $\begin{array}{l}\max \text { util. } \\
{[-]}\end{array}$ & $\begin{array}{l}\mathrm{EE} \\
{[\mathrm{MJ}]}\end{array}$ & $\begin{array}{l}\mathrm{EC} \\
{\left[\mathrm{kgCO}_{2} \mathrm{eq}\right]}\end{array}$ \\
\hline A & $(\mathrm{P})+\mathrm{G}_{\mathrm{opt}}$ & 3'840 & 65 & 63 & $53 \%$ & $90 \%$ & $12^{\prime} 700$ & 1'080 \\
\hline B & (P) $+\mathrm{G}_{\mathrm{opt}}$ & 4'950 & 127 & 52 & $45 \%$ & $90 \%$ & $16 ' 500$ & 1'400 \\
\hline $\mathrm{C}$ & (P) $+\mathrm{G}_{\text {opt }}$ & 5,370 & 204 & 70 & $41 \%$ & $89 \%$ & $18^{\prime} 100$ & 1'540 \\
\hline New & & 2’090 & - & - & $54 \%$ & $100 \%$ & $27^{\prime} 600$ & 1'930 \\
\hline
\end{tabular}

In a post process step, an FE analysis including geometric compatibility showed that 19 members in the minimum weight structure and up to 28 members in the reuse cases are overstressed. In practice, overstressed members could be replaced and members prone to buckling could be braced.

\subsection{Stock synthesis for three kit-of-parts structures}

This section shows the application of the stock optimization formulation (S) to build three truss structures of different topology: an arch, a portal and a Howe truss, Figure 7. Multiple load cases are applied to each structure and all standard circular hollow sections of EN 10210 are assumed to be available. The inner nodes of the arch and the portal are constrained in the geometry optimization to maintain a minimum height of 4.0 and $8.0 \mathrm{~m}$, respectively. A maximum number of $z^{\max }=12$ unique element types (length and cross section) is allowed.

(a)

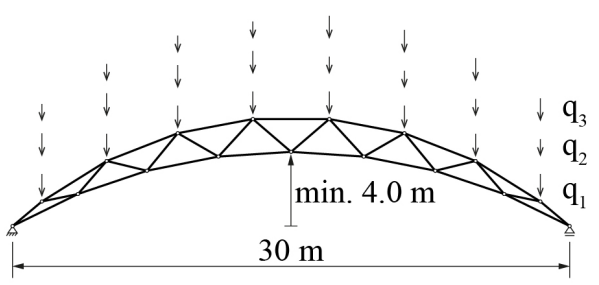

(b)

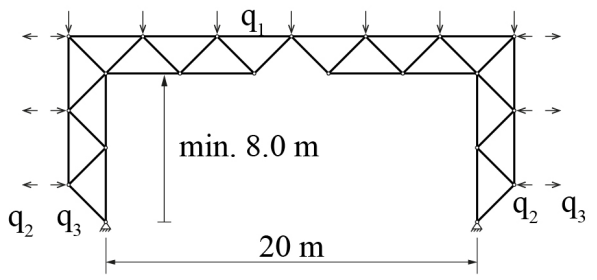

(c)

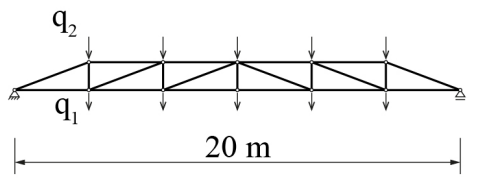

Figure 7: Arch, portal and Howe truss
When solving (S), the optimization of $\mathcal{R}$ structures (in this case 3) must be carried out simultaneously, resulting in a large number of optimization variables. To reduce computation time, geometric compatibility is omitted in this case study. However, because the three systems are statically determinate, all member forces are correctly obtained through force equilibrium only and thus members will not be overstressed.

(a)

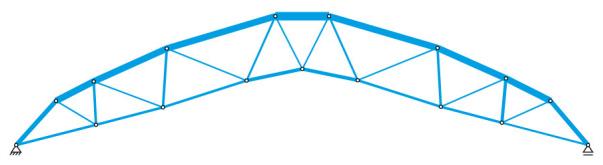

(b)

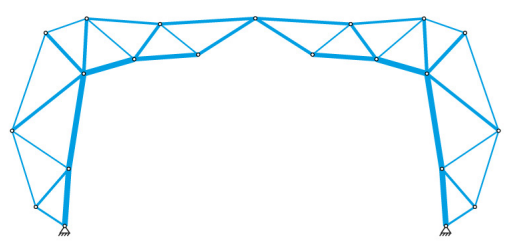

(c)

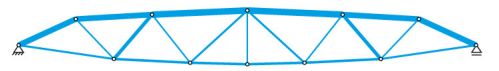

Figure 8: One-off minimum weight solutions

Figure 8 shows the one-off minimum weight solutions for each individual structure, irrespectively of the common stock. Figure 9(a) shows the three optimized layouts, which share a common kit of parts. In Figure 9 each member color corresponds to one of the twelve element types in the kit of parts. The bar charts in Figure 9(b) indicate cross section size, element length and assignment. In total 40 kitof-parts elements are required to build all three systems (90 members in total). The elements of group 11 are reused in all three systems and have a large cross section due to buckling constraints. The elements of group 2 are uniquely used for the portal case. 
(a)

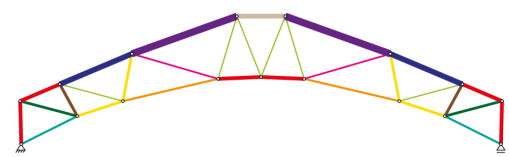

(b)

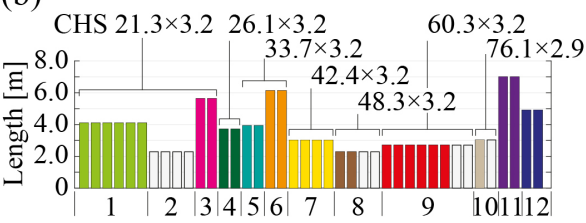

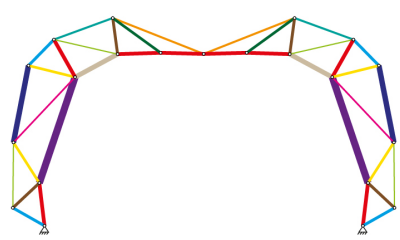
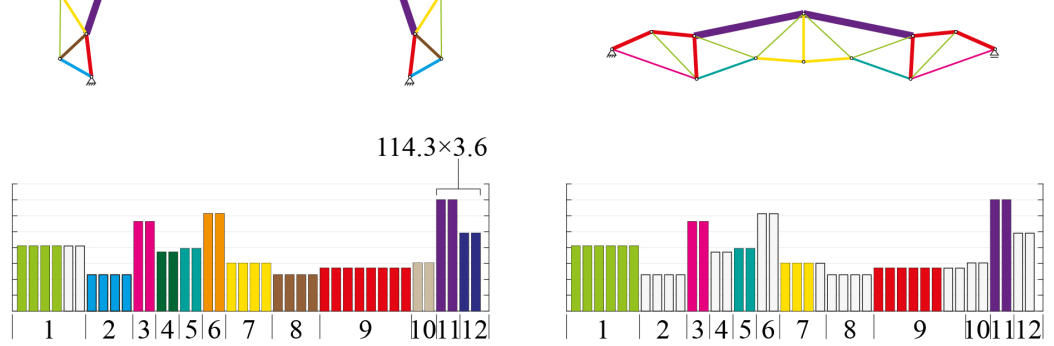

Figure 9: Optimal kit-of-parts design: a) optimal geometries and element assignment, b) element assignment and stock description, ordered by cross-section size

The elements of group 12 are under tension in the portal case but in compression in the arch. Elements in groups 2 and 8 as well as in 7 and 10 have identical lengths. By using their respectively larger cross section these groups could be merged to reduce the number of different element types at a cost of an increase of the structural weight.

Table 4: Results for the minimum weight systems

\begin{tabular}{llllll}
\hline & & Arch & Portal & Howe & Sum \\
\hline Members & {$[-]$} & 31 & 38 & 21 & $\mathbf{9 0}$ \\
Mass & {$[\mathrm{kg}]$} & 429 & 385 & 256 & $\mathbf{1 ' 0 7 0}$ \\
mean util. & {$[-]$} & $81 \%$ & $83 \%$ & $74 \%$ & $\mathbf{8 0 \%}$ \\
max util. & {$[-]$} & $100 \%$ & $100 \%$ & $100 \%$ & $\mathbf{1 0 0 \%}$ \\
EE & {$[\mathrm{MJ}]$} & $5{ }^{\prime} 670$ & 5,090 & $3{ }^{\prime} 390$ & $\mathbf{1 4}$ '150 \\
EC & {$\left[\mathrm{kgCO}_{2} \mathrm{eq}\right] 397$} & 356 & 237 & $\mathbf{9 9 0}$ \\
\hline
\end{tabular}

Table 5: Results for the kit of parts

\begin{tabular}{llllll}
\hline & & Arch & Portal & Howe & $\begin{array}{l}\text { Kit of } \\
\text { parts }\end{array}$ \\
\hline Members & {$[-]$} & - & - & - & $\mathbf{4 0}$ \\
Mass & {$[\mathrm{kg}]$} & 490 & 548 & 309 & $\mathbf{5 6 0}$ \\
mean util. & {$[-]$} & $61 \%$ & $60 \%$ & $68 \%$ & $\mathbf{6 2 \%}$ \\
max util. & {$[-]$} & $96 \%$ & $99 \%$ & $97 \%$ & $\mathbf{9 9 \%}$ \\
EE & {$[\mathrm{MJ}]$} & - & - & - & $\mathbf{7}, 410$ \\
EC & {$\left[\mathrm{kgCO}_{2} \mathrm{eq}\right]-$} & - & - & $\mathbf{5 2 0}$ \\
\hline
\end{tabular}

Table 4 and 5 give optimization metrics for the minimum weight systems and the optimal kit of parts respectively. Because kit-of-parts elements are reused in multiple structures, they need to be sized for the worst loading condition, i.e. reused elements are oversized and have a lower mean capacity utilization (util.) compared to the one-off minimum weight solutions. However, producing only 40 instead of 90 elements reduces environmental impacts significantly by $48 \%$ in terms of embodied energy and carbon equivalence. A post process FE analysis showed that typical SLS displacement limits are satisfied.

\section{DISCUSSION}

The proposed formulations based on mixed-integer linear programming produce globally optimal results and are applicable to small and medium-scale problems without simplifications. Large-scale problems might not converge in a reasonable time. Simplifications including omission of geometric compatibility and symmetry constraints had to be made in this work.

Connection design was out of the scope of this paper. However, the proposed methods are applicable to typical truss topologies and modular space trusses. In addition, the geometry optimization could be guided towards user-defined design features or to account for available joint types.

\section{CONCLUSION}

The case studies presented in this work have shown that novel and diverse structure layouts are obtained when structural elements are reused for multiple service cycles i.e. when form follows availability. The methods outlined in this paper allow for a substantial reduction of environmental impacts of load-bearing systems. Topology and geometry optimization subject to stock availability allows to account for environmental considerations at early stages of the design process. Design through reuse of stock elements therefore opens up new directions in structural form finding and optimization. 
The case studies show that stock variability largely influences design outcomes. Future work could apply the method to real inventories of elements in order to generalize the conclusions reached in this paper. Future work could also look into studying the influence of conditions and residual life of reclaimed elements on design outcomes.

Optimization of the stock has shown to be a promising approach to design multiple structures from a common kit of parts. This method could be employed to design temporary structures that adapt to several sites or generally to reuse structural elements over multiple service cycles. Future research could study further the effect of the weighting for the multi-objective kit-of-parts optimization problem.

\section{REFERENCES}

[1] M. Gorgolewski, "Designing with reused building components: some challenges," Build. Res. Inf., vol. 36, no. 2, pp. 175-188, 2008. (DOI: 10.1080/09613210701559499)

[2] M. Gorgolewski, Resource Salvation: The Architecture of Reuse. John Wiley \& Sons, 2017. (DOI: 10.1002/9781118928806)

[3] Y. Fujitani and D. Fujii, "Optimum structural design of steel plane frame under the limited stocks of members," in Proceedings of the RILEM/CIB/ISO International Symposium, Integrated Life-Cycle Design of Materials and Structures, 2000, pp. 198-202.

[4] A. Bukauskas, P. Shepherd, P. Walker, B. Sharma, and J. Bregula, "Form-Fitting strategies for diversity-tolerant design," in Proceedings of the IASS Annual Symposium, Hamburg, 2017.

[5] W. Nadir, I. Y. Kim, D. Hauser, and O. de Weck, "Multidisciplinary structural truss topology optimization for reconfigurability," in 10th AIAA/ISSMO Multidisciplinary Analysis and Optimization Conference, Albany, 2004. (DOI: 10.2514/6.2004-4337)

[6] A. Tugilimana, A. P. Thrall, and R. Filomeno Coelho, "Conceptual Design of Modular Bridges Including Layout Optimization and Component Reusability," J. Bridge Eng., vol. 22, no. 11, p. 04017094, 2017. (DOI: 10.1061/(ASCE)BE.1943-5592.0001138)

[7] P. Basso, A. E. Del Grosso, A. Pugnale, and M. Sassone, "Computational morphogenesis in architecture: Cost optimization of free-form grid shells," J. Int. Assoc. Shell Spat. Struct., vol. 50, no. 3, pp. 143-150, 2009.

[8] O. N. Ghattas and I. E. Grossmann, "MINLP and MILP Strategies for Discrete Sizing Structural Optimization Problems," in Electronic Computation, 1991, pp. 197-204.

[9] J. Brütting, J. Desruelle, G. Senatore, and C. Fivet, "Design of Truss Structures Through Reuse," Structures, vol. 18, pp. 128-137, 2019. (DOI: 10.1016/j.istruc.2018.11.006)

[10] R. T. Haftka, "Simultaneous analysis and design," AIAA J., vol. 23, no. 7, pp. 10991103, 1985. (DOI: 10.2514/3.9043) 